\title{
Impact of Age on Surgical Outcomes after Robot Assisted Laparoscopic Hysterectomies
}

\author{
A. Eddib ${ }^{1}$, S. Hughes ${ }^{1,2}$, M. Aalto ${ }^{1}$, A. Eswar ${ }^{1}$, M. Erk¹, C. Michalik ${ }^{3}$, V. Krovi' ${ }^{4}$, P. Singhal1 \\ ${ }^{1}$ Department of Obstetrics and Gynecology, University at Buffalo, Buffalo, USA \\ ${ }^{2}$ Department of Robotic Surgery, Williamsville, USA \\ ${ }^{3}$ Kaleida Health, Millard Fillmore Suburban Hospital, Williamsville, USA \\ ${ }^{4}$ Department of Mechanical and Aerospace Engineering, University at Buffalo, Buffalo, USA \\ Email: abeereddib@hotmail.com
}

Received 25 December 2013; revised 20 January 2014; accepted 28 January 2014

Copyright (C) 2014 by authors and Scientific Research Publishing Inc.

This work is licensed under the Creative Commons Attribution International License (CC BY).

http://creativecommons.org/licenses/by/4.0/

(c) (i) Open Access

\section{Abstract}

Objective: To estimate the impact of patient's age on surgical outcomes in patients undergoing robotic hysterectomy. Methods: A retrospective review of prospectively collected cohort data for a consecutive series of patients undergoing gynecologic robotic surgery. Patient's age and perioperative variables were collected from the database, charts, and other hospital records of all patients undergoing robotic hysterectomy. Results: 399 patients underwent robotic surgery for gynecologic disease. 370 patients who were under age 70 were compared with 29 patients who were over age 70 . When comparing all patients under age 70 with patients over age 70 , the mean age was 48.4 and $77(P<0.05)$, mean BMI was 32.1 and $28.3 \mathrm{~kg} / \mathrm{m}^{2}(P<0.05)$, mean procedure time was 185 and 211 minutes $(\mathrm{min})(\mathrm{P}=0.09)$, mean console time was 123 and $148 \mathrm{~min}(\mathrm{P}=$ 0.056), mean OR (Operating room) time was 237 and 273, mean EBL (Estimated blood loss) was 71 and $65 \mathrm{ml}(\mathrm{P}=0.74), \mathrm{Hb}($ Hemoglobin) drop was 1.4 and $1.2(P=0.45)$, uterine weight was 212 and $95 \mathrm{gm}(P=0.98)$, and length of stay was 1.4 and 1.6 days $(P=0.33)($ Table 1$)$. The patients over age 70, when procedures were combined, had a statistically significant lower mean BMI, uterine weight and longer Operating room (OR) time. However, when stratified by the type of procedure performed, there was no difference in surgery times among those under $\mathbf{7 0}$ and over $\mathbf{7 0}$ years of age. The elderly patients were more likely to have cancer, which was in almost half the elderly patients, and thus necessitate staging. Thus adding the performance of lymph node dissection likely resulted in the increased length of the surgery time that was noted in the combined group (Tables 1, 2). There were no operative deaths. Conclusions: Advanced age does not appear to be associated with an increased risk of morbidity, or adverse perioperative outcomes in patients undergoing robotic hysterectomy.

*Corresponding author. 


\section{Keywords}

\section{Robotics; Surgery; Age; Hysterectomy}

\section{Introduction}

Minimally invasive surgical methods are becoming more common in the treatment of gynecologic conditions, both benign and malignant diseases. There is sufficient evidence that minimally invasive surgery can reduce perioperative blood loss, blood transfusions, postoperative pain, complications, recovery time, and hospital stay [1]-[5]. However, despite the documented benefits of the minimally invasive approach in patients, due to the steep learning curve, and technical difficulties, this has been slow to gain acceptance [6]. More than two thirds of hysterectomies are being performed through an abdominal incision [7].

With the introduction of robotic-assisted laparoscopic surgery, the percentage of patients that undergo a minimally invasive approach has increased. Robotic-assisted surgery as compared to traditional laparoscopy offered better ergonomics, increased dexterity, as well as improved visualization via 3-dimensional high-definition camera systems. This increase in use of robotic-assisted surgery brings into question the safety of its use in our patients. Concerns about patient safety in robotic-assisted surgery arise when technical aspects of the procedure are taken into consideration. Steep (30 - 40 degrees) Trendelenburg positioning can affect the cardiovascular and respiratory systems and require higher inspiratory pressures [8]. In addition, it is thought that time spent in the Operating room may be increased due to time spent in positioning the robot and placing trocars [3] [9]. Taken together, these aspects of robotic-assisted surgery, along with medical comorbidities, and physiologic changes inherent in the geriatric population put into question its safety in our elderly patients.

The elderly population has become an increasing fraction of the total population in the United States. Currently, the "baby boomer" generation, people born between 1946 and 1964, started to reach the age of 65 in 2011 and accounts for approximately 20\% of the total US population [10]. Additionally, this older population aged 65 and older is projected to double in number in 2030 (census, 2006).

With advancing age come medical comorbidities including, hypertension, peripheral vascular disease, diabetes, chronic pulmonary disease, arthritis, and neurological conditions [8] [11]. These diseases increase the risk of general anesthesia. Due to the increase in life expectancy throughout the world, older patients that will be presenting for surgical procedures will continue to grow. There are studies that suggest that traditional laparoscopy in elderly patients is safe, and effective with acceptable perioperative outcomes. However, information about the impact of age on robotic surgery in gynecology has been limited. Robotic assisted laparoscopic surgery involves both the use of extreme positioning and pneumoperitoneum. Each of these can lead to hemodynamic changes, which can be detrimental in an already compromised elderly population. These types of studies are necessary because elderly patients are being managed based on assumptions deduced from younger populations [12]. The objective of the present study was to compare the differences in perioperative outcomes between elderly women ( $\geq 70$ years old) and younger women ( $<70$ years old). This study has been approved by the University at Buffalo, The State University of New York, Health Science Institutional Review Board with an HSIRB project\# GYN0521011E.

\section{Methods}

This was a retrospective review of prospectively collected data on a consecutive series of robotic assisted gynecologic surgeries in a single institution from May 2010 till May 2012. The University at Buffalo Health Sciences Institutional Review Board approved the study. Robotic surgeries were performed on the da Vinci Surgical System (Intuitive Surgical, Sunnydale, CA) using a four-arm robot. The study population includes a consecutive series of robot assisted gynecologic surgeries performed by approximately 10 surgeons with different experience levels. Of these surgeons, one had prior experience at another institution and the remaining surgeons were all beginners and had no prior experience. Thus the majority of cases in our cohort consisted surgeons in the early part of the learning curves. The types of procedures were Robotic Assisted Hysterectomy with or without Bilateral Salpingo-oophorectomy (RAH +/- BSO), and RoboticHysterectomies with Lymph Node Dissection, with 
or without Bilateral Salpingo-oophorectomy (RAH + LND +/- BSO). The patients were grouped and analyzed into three cohorts based on type of procedure performed. Cohort 1 (Table 1) included all robotic hysterectomy patients $\{(\mathrm{RAH}+/-\mathrm{BSO})$ and $(\mathrm{RAH}+\mathrm{LND}+/-\mathrm{BSO})\}$, which included the (RAH $+/-\mathrm{BSO})$ cohort and the (RAH + LND +/- BSO) cohort. Then we stratified this cohort into a cohort with staging lymphadenectomy and one without. Thus, Cohort 2 was (RAH+/- BSO) (Table 2) and Cohort 3 was (RAH + LND +/- BSO) (Table $3)$. In each group the patients under age 70 were compared with patients aged 70 and over, in terms of perioperative variables and outcomes. All variables were collected prospectively from the OR records, and patient's chart. Patient characteristics such as age and BMI, and perioperative variables were collected. Perioperative variables included estimated blood loss (EBL), Hb (Hemoglobin) drop, length of hospital stay (LOS), uterine weight (ut wt.), and complications. Operative times and duration of each step were also recorded and included: mean total operative time (from patient in Operating room (OR) till patient out of OR), prep time (patient asleep to skin incision), time to dock, console time, closing time (from undocking till port site fascia closure), and procedure time (skin incision to dressing). Complications we considered significant and included in our study were those that were intraoperative or postoperative. The postoperative complications either occurred prior to discharge or necessitated readmission to the hospital. The complications reported were collected from patient charts and included a hospital wide electronic query of any patients re-admitted within 30 days of the surgery. Data analysis was performed using SAS 9.3 (Cary, NC). Descriptive statistics were initially performed followed by statistical analysis with Student's t test to compare means between two groups. Significance was set at $\mathrm{P}<0.05$.

\section{Results}

A total of 399 patients underwent robotic surgery for Gynecologic disease. Of these, 370 patients that were under age 70 were compared with 29 patients who were over age 70 . When comparing all patients under age 70 with patients over age 70, in cohort 1 (hysterectomy with and without lymphadenectomy) \{(RAH +/- BSO) and $(\mathrm{RAH}+\mathrm{LND}+/-\mathrm{BSO})\}$, the mean age was 48.4 and $77(\mathrm{P}<0.05)$, mean BMI was 32.1 and $28.3 \mathrm{~kg} / \mathrm{m}^{2}(\mathrm{P}<$ 0.05), mean procedure time was 185 and $211 \mathrm{~min}(\mathrm{P}=0.09)$, mean console time 123 and $148 \mathrm{~min}(\mathrm{P}=0.056)$, mean OR (Operating room) time 237 and 273, mean EBL (Estimated blood loss) was 71 and $65 \mathrm{ml}(\mathrm{P}=0.74)$, $\mathrm{Hb}$ drop 1.4 and $1.2(\mathrm{P}=0.45)$, uterine weight was 212 and $95 \mathrm{gm}(\mathrm{P}=0.98)$, and length of stay was 1.4 and 1.6 days $(\mathrm{P}=0.33)$, respectively (Table 1$)$.

The patients over age 70 when combined had a statistically significant lower mean BMI, uterine weight and longer Operating room (OR) time. Therefore, we decided to stratify cohort 1 into cohort 2 (RAH +/- BSO) and

Table 1. Demographic and perioperative variables in patients that underwent a robotic hysterectomy with or without lymph node dissection combined (RAH + RAH/LND).

\begin{tabular}{cccc}
\hline Variable & Age $<70$ & Age $>70$ & P Value \\
N & 370 & 29 & $<0.05$ \\
Age & 48.4 & 77 & $<0.05$ \\
BMI & 32.1 & 28.3 & 0.74 \\
EBL & 71.4 & 65.1 & 0.45 \\
Hb drop & 1.4 & 1.2 & 0.33 \\
LOS & 1.42 & 1.6 & $<0.05$ \\
Utwt & 212.8 & 95 & 0.73 \\
Time to dock & 28.7 & 30.1 & 0.056 \\
Console time & 123.5 & 148.7 & 0.14 \\
Prep time & 11.2 & 9.4 & 0.38 \\
Closing time & 34.3 & 31.3 & 0.09 \\
Procedure time & 185.9 & 211.8 & $<0.05$ \\
OR time & 237 & 273 & $1(3 \%)$ \\
Complications & $23(6 \%)$ & & - \\
\hline
\end{tabular}


Table 2. Demographic and perioperative variables in patients that underwent a robotic hysterectomy with or without adnexal surgery, but no lymph node dissection (RAH).

\begin{tabular}{cccc}
\hline Variable & Age $<70$ & Age $>70$ & P Value \\
\hline N & 302 & 12 & $<0.05$ \\
Age & 46.9 & 76.4 & $<0.05$ \\
BMI & 31.7 & 27.4 & 0.7 \\
EBL & 73.09 & 91.11 & 0.9 \\
Hb drop & 1.47 & 1.48 & 0.5 \\
LOS & 1.3 & 1.14 & $<0.05$ \\
Utwt & 218.7 & 90.7 & 0.24 \\
Time to dock & 28.9 & 23 & 0.84 \\
Console time & 112.5 & 116 & $<0.05$ \\
Prep time & 11.3 & 7.3 & 0.54 \\
Closing time & 32.9 & 30 & 0.83 \\
Procedure time & 173.7 & 169 & 0.79 \\
OR time & 224.6 & 232 & - \\
Complications & $17(5 \%)$ & $1(8 \%)$ & \\
\hline
\end{tabular}

Table 3. Demographic and perioperative variables in patients that underwent a robotic hysterectomy with staging lymph node dissection (RAH/LND).

\begin{tabular}{cccc}
\hline Variable & Age $<70$ & Age $>70$ & P Value \\
\hline N & 45 & 14 & $<0.05$ \\
Age & 57 & 77.5 & $<0.05$ \\
BMI & 34.4 & 28.8 & 0.36 \\
EBL & 62.9 & 51.7 & 0.98 \\
Hb drop & 0.87 & 0.87 & 0.8 \\
LOS & 1.7 & 1.8 & 0.16 \\
Utwt & 144.7 & 112.1 & 0.28 \\
Time to dock & 28 & 33.6 & 0.9 \\
Console time & 174.8 & 172 & 0.27 \\
Prep time & 11.6 & 9.1 & 0.31 \\
Closing time & 39.1 & 32.3 & 0.85 \\
Procedure time & 243.6 & 239.1 & 0.89 \\
OR time & 301.7 & 298.4 & - \\
Complications & $6(13 \%)$ & 0 & \\
\hline
\end{tabular}

cohort 3 (RAH + LND +/- BSO), by type of procedure performed to determine if the difference in length of procedure could be explained by type of procedure distribution between the age groups.

In cohort 2 (RAH +/- BSO) (Table 2), the only statistically significant differences noted were the lower BMI and lower uterine weights among the older patients, with a longer prep time. In cohort 3 (RAH + LND +/- BSO) (Table 3), the only statistically significant difference noted was in BMI, with no difference in operative times. There were no operative deaths in either group. The total complication rate was 6\%, with 23 complications in the under 70 (6\%), and one complication (1\%) in the over 70 age group. The complication in the over 70 age 
group was an ICU admission for a difficult intubation. The less than 70 age group complication varied, and included 2 intraoperative conversions, one was to deliver an intact specimen, and the other was for extensive adhesions. Also, five patients returned to the OR for the following reasons: a strangulated umbilical hernia necessitating a bowel resection, a vaginal cuff dehiscence, a ureteral transection, bowel injury, and a vaginal laceration. Other non-operative (not requiring return to the OR) complications included: ileus (4), transfusion (2), pelvic abscesses (3), respiratory (Non-Pulmonary embolism) issues (2), cellulitis (1), intraoperative bladder injury and repair (1), pulmonary embolism (1), ureteral thermal injury (1), and myositis (1). It appears that the older population seemed to have less complications, furthermore, when stratified by the type of procedure performed, there was no difference in surgery times among those under 70 and over 70 years of age (Table 1).

\section{Discussion}

The benefit of the minimally invasive approach is well documented, as it results in less blood loss, less abdominal wall infections, shorter hospitalization, and quicker recovery. For the elderly, these effects translate into a quicker return of independence after surgery, and better quality of life. However, due to the steep learning curve, and technical difficulties, traditional laparoscopy has been slow to gain acceptance. With the recent adoption of the da Vinci Surgical System for robotic surgery, surgeons have been able to overcome many of the limitations of traditional laparoscopy. Benefits of robotic surgery include 3-dimensional high-definition visualization, improved surgeon ergonomics, wristed instruments, increased dexterity and surgical precision.

The elderly patient presents a different set of challenges for the gynecologic surgeon. Co-morbidities prevalent in this population, such as, hypertension, peripheral vascular disease, diabetes, chronic pulmonary disease, arthritis, and neurological conditions may expose the patient to risk of serious morbidities and even death [8] [11]. Other additional risks unique to the elderly patient are intraoperative thermoregulation, volume depletion and overload, as well as deep vein thrombosis [13].

Patient positioning in both laparoscopic and robotic-assisted MIS is an important factor to consider in the elderly population. Elderly patients placed in dorsal lithotomy position for gynecologic procedures to allow for increased surgical access, and uterine manipulation may be exposed to risk of injury. Osteoarthritis, osteoporosis, and joint prosthesis inherent in the elderly population may limit patient positioning and lead to postoperative neuropathy, or injury [13]. In addition to the dorsal lithotomy position, steep Trendelenburg (30 - 40 degrees) is utilized to perform laparoscopic and robotic-assisted MIS. Steep Trendelenburg positioning allows for the abdominal contents to be displaced into the upper abdomen allowing for better visualization of the pelvis and decreased injury in gynecologic surgery. In a study of cardiac function during robotic surgery, deep trendelenburg position was reported to increase stroke volume [14], and result in the need for higher inspiratory pressures [15].

Pneumoperitoneum (15 - $20 \mathrm{mmHg}$ ) is also employed and has a number of physiologic consequences, leading to an increase in mean arterial pressure [8] [16], systemic vascular resistance [8] [16] and inferior vena caval pressure [8]. The combination of steep Trendelenburg and pneumoperitoneum, used in robotic-assisted MIS has been shown increase mean arterial blood pressure, as well as right and left ventricular filling pressures, and pulmonary capillary wedge pressure in both healthy patients and in those with cardiopulmonary disease [17]. In addition, pneumoperitoneum and head-down tilt have both been found to induce intracranial hypertension [18], and in elderly patients to reduce cerebral tissue oxygen saturation [19]. Whether or not these physiologic changes can lead to cardiovascular or neurologic injury in elderly patients undergoing long robotic-assisted MIS procedures has not been well established. However, more recently, it was found that prolonged steep Trendelenburg and pneumoperitoneum was tolerated well and that regional cerebral oxygenation was preserved in male patients undergoing Robotic-assisted laparoscopic radical prostatectomies [20]. Other risks of steep Trendelenburg also involve possible injury to the eye, even blindness, as intraocular pressures are elevated for long durations of robotic-assisted MIS [21] [22]. The elderly are at an increased risk as they have a higher incidence of pre-existing eye disease. It has been suggested that ophthalmic consultation be considered, and that the risks of ophthalmic injury be discussed with the elderly patient prior to surgery.

Currently, in the field of gynecology, only one other study looks into the differences between the elderly and younger patients undergoing robotic-assisted MIS [23]. The study found that despite the elderly group having a significantly higher rate of comorbidities, mean operative time and blood loss during surgery were similar as compared to the younger group [23]. There was a trend towards the elderly patient having an increased hospital stay as compared to the younger group but was not statistically significant. Our study had similar findings, as we 
also found that mean blood loss, and operative times were similar when stratified by type of surgery, i.e. hysterectomy with lymph node dissection. Since patients over age 70, when types of procedures were combined, had a statistically significant lower mean BMI, uterine weight and longer Operating room (OR) time we decided to stratify the patients by type of procedure. Especially since the elderly patients in our study were found to have an increased rate of cancer, and underwent staging. The addition of a lymphadenectomy as part of their staging, likely explains the increase in operative time in the combined procedures group. The elderly patients were more likely to have cancer, which was in almost half the elderly patients, and thus necessitated staging. Thus adding the performance of lymph node dissection likely resulted in the increased length of the surgery time that was noted in the combined procedures group (Cohort 1, RAH and RAH/LND).

In cohort 2 (RAH +/- BSO) (Table 2), the only statistically significant differences noted were the lower BMI and lower uterine weights among the older patients, with a longer prep time. These differences in BMI and uterine weights, could account for the longer prep time, since heavier patients could take more time to position and prep. In cohort 3 (RAH + LND +/- BSO) (Table 3), the only statistically significant difference noted was in BMI, with no difference in operative times.

Weaknesses of our study include the low numbers of women over 70 years old. Our study used an age cutoff that is higher than the traditional age 65 as we wanted to see the impact on this group to provide a significant age contrast, consequently resulting in a small sample size. Another limitation of our study is the lack of information on outpatient follows up. We only included hospital based complications either at the index admission or a later re-admission. Even though we do not think the impact on our conclusion would be significant since those complications that did not require hospital admission are more likely to be associated with lower morbidity; it is nonetheless a limitation.

\section{Conclusion}

Our study demonstrates that advanced age does not appear to be associated with an increased risk of morbidity, or adverse perioperative outcomes in patients undergoing gynecologic robotic-assisted minimally invasive surgery. Additional research should be performed in the elderly to ensure safety of minimally invasive surgery for this expanding population.

\section{Disclosure}

The authors have no conflict of interest to report.

\section{References}

[1] Lee, M., et al. (2011) Comparisons of Surgical Outcomes, Complications, and Costs between Laparotomy and Laparoscopy in Early-Stage Ovarian Cancer. International Journal of Gynecological Cancer, 21, 251-256. http://dx.doi.org/10.1097/IGC.0b013e318208c71c

[2] Kalogiannidis, I., et al. (2007) Laparoscopy-Assisted Vaginal Hysterectomy Compared with Abdominal Hysterectomy in Clinical Stage I Endometrial Cancer: Safety, Recurrence, and Long-Term Outcome. American Journal of Obstetrics \& Gynecology, 196, e1-8.

[3] Nezhat, C., et al. (2009) Laparoscopic Hysterectomy with and without a Robot: Stanford Experience. JSLS, 13, $125-128$.

[4] Soto, E., et al. (2011) Total Laparoscopic Hysterectomy versus da Vinci Robotic Hysterectomy: Is Using the Robot Beneficial? Journal of Gynecologic Oncology, 22, 253-259. http://dx.doi.org/10.3802/jgo.2011.22.4.253

[5] Payne, T.N. and Dauterive, F.R. (2008) A Comparison of Total Laparoscopic Hysterectomy to Robotically Assisted Hysterectomy: Surgical Outcomes in a Community Practice. Journal of Minimally Invasive Gynecology, 15, $286-291$. http://dx.doi.org/10.1016/j.jmig.2008.01.008

[6] Wu, J.M., et al. (2007) Hysterectomy Rates in the United States, 2003. Obstetrics \& Gynecology, 110, $1091-1095$. http://dx.doi.org/10.1097/01.AOG.0000285997.38553.4b

[7] Merrill, R.M. (2008) Hysterectomy Surveillance in the United States, 1997 through 2005. Medical Science Monitor, 14, CR24-31.

[8] Efron, D.T. and Bender, J.S. (2001) Laparoscopic Surgery in Older Adults. Journal of the American Geriatrics Society, 49, 658-663. http://dx.doi.org/10.1046/j.1532-5415.2001.49130.x

[9] Nezhat, C., et al. (2009) Robot-Assisted Laparoscopic Surgery in Gynecology: Scientific Dream or Reality? Fertility 
and Sterility, 91, 2620-2622. http://dx.doi.org/10.1016/j.fertnstert.2008.03.070

[10] Yoshikawa, T.T. (2012) Future Direction of Geriatrics: “Gerogeriatrics”. Journal of the American Geriatrics Society, 60, 632-634. http://dx.doi.org/10.1111/j.1532-5415.2012.03896.x

[11] Leung, J.M. and Dzankic, S. (2001) Relative Importance of Preoperative Health Status versus Intraoperative Factors in Predicting Postoperative Adverse Outcomes in Geriatric Surgical Patients. Journal of the American Geriatrics Society, 49, 1080-1085. http://dx.doi.org/10.1046/j.1532-5415.2001.49212.x

[12] Ramesh, H.S., et al. (2005) Optimising Surgical Management of Elderly Cancer Patients. World Journal of Surgical Oncology, 3, 17. http://dx.doi.org/10.1186/1477-7819-3-17

[13] Atiemo, H., Griebling, T.L. and Daneshgari, F. (2006) Advances in Geriatric Female Pelvic Surgery. BJU International, 98, 90-96. http://dx.doi.org/10.1111/j.1464-410X.2006.06301.x

[14] Lau, S., et al. (2011) Relationship between Body Mass Index and Robotic Surgery Outcomes of Women Diagnosed with Endometrial Cancer. International Journal of Gynecological Cancer, 21, 722-729. http://dx.doi.org/10.1097/IGC.0b013e318212981d

[15] Vaknin, Z., et al. (2010) Outcome and Quality of Life in a Prospective Cohort of the First 100 Robotic Surgeries for Endometrial Cancer, with Focus on Elderly Patients. International Journal of Gynecological Cancer, 20, 1367-1373.

[16] Falabella, A., et al. (2007) Cardiac Function during Steep Trendelenburg Position and $\mathrm{CO}_{2}$ Pneumoperitoneum for Robotic-Assisted Prostatectomy: A Trans-Oesophageal Doppler Probe Study. International Journal of Medical Robotics, 3, 312-315. http://dx.doi.org/10.1002/rcs.165

[17] Lestar, M., et al. (2011) Hemodynamic Perturbations during Robot-Assisted Laparoscopic Radical Prostatectomy in 45 Degrees Trendelenburg Position. Anesthesia \& Analgesia, 113, 1069-1075. http://dx.doi.org/10.1213/ANE.0b013e3182075d1f

[18] Halverson, A., et al. (1998) Evaluation of Mechanism of Increased Intracranial Pressure with Insufflation. Surgical Endoscopy, 12, 266-269. http://dx.doi.org/10.1007/s004649900648

[19] Casati, A., et al. (2007) Monitoring Cerebral Oxygen Saturation in Elderly Patients Undergoing General Abdominal Surgery: A Prospective Cohort Study. European Journal of Anaesthesiology, 24, 59-65.

[20] Kalmar, A.F., et al. (2010) Influence of Steep Trendelenburg Position and CO(2) Pneumoperitoneum on Cardiovascular, Cerebrovascular, and Respiratory Homeostasis during Robotic Prostatectomy. British Journal of Anaesthesia, 104, 433-439. http://dx.doi.org/10.1093/bja/aeq018

[21] Awad, H., et al. (2009) The Effects of Steep Trendelenburg Positioning on Intraocular Pressure during Robotic Radical Prostatectomy. Anesthesia \& Analgesia, 109, 473-478. http://dx.doi.org/10.1213/ane.0b013e3181a9098f

[22] Weber, E.D., et al. (2007) Posterior Ischemic Optic Neuropathy after Minimally Invasive Prostatectomy. Journal of Neuro-Ophthalmology, 27, 285-287. http://dx.doi.org/10.1097/WNO.0b013e31815b9f67

[23] Vaknin, Z., et al. (2010) Outcome and Quality of Life in a Prospective Cohort of the First 100 Robotic Surgeries for Endometrial Cancer, with Focus on Elderly Patients. International Journal of Gynecological Cancer, 20, 1367-1373.

\section{Abbreviations}

RAH: Robotic Assisted Hysterectomy

BSO: Bilateral Salpingo-oophorectomy

LND: Lymph Node Dissection 